\title{
Téoros
}

Revue de recherche en tourisme

\section{Le transport aérien}

\section{Le voyage à bilan carbone neutre est-il réellement écologique ?}

\section{Julianna Priskin}

Volume 26, numéro 1, printemps 2007

URI : https://id.erudit.org/iderudit/1070997ar

DOI : https://doi.org/10.7202/1070997ar

Aller au sommaire du numéro

Éditeur(s)

Université du Québec à Montréal

ISSN

0712-8657 (imprimé)

1923-2705 (numérique)

Découvrir la revue

\section{Citer cette note}

Priskin, J. (2007). Le transport aérien : le voyage à bilan carbone neutre est-il réellement écologique ? Téoros, 26(1), 63-66. https://doi.org/10.7202/1070997ar

Ce document est protégé par la loi sur le droit d'auteur. L'utilisation des services d'Érudit (y compris la reproduction) est assujettie à sa politique d'utilisation que vous pouvez consulter en ligne.

https://apropos.erudit.org/fr/usagers/politique-dutilisation/
Cet article est diffusé et préservé par Érudit.

Érudit est un consortium interuniversitaire sans but lucratif composé de l’Université de Montréal, l'Université Laval et l'Université du Québec à Montréal. Il a pour mission la promotion et la valorisation de la recherche. https://www.erudit.org/fr/ 


\section{Réseau de veille en tourisme - Chaire de tourisme Transat}

\section{Le transport aérien \\ Le voyage à bilan carbone neutre est-il réellement écologique?}

\begin{abstract}
Julianna Priskin
L'industrie du voyage contribue au changement climatique anthropique dans la mesure où elle implique la consommation de biens et services qui nécessitent l'utilisation de combustibles fossiles, tant durant le trajet qu'au point de destination. Cette consommation entraîne des émissions de gaz à effet de serre $(G E S)^{1}$ dont les répercussions environnementales, directes et indirectes, continuent d'être omniprésentes (IPCC, 2007). Bien que le tourisme contribue aux émissions de GES à un degré moindre que d'autres secteurs, il est plus que temps de sonner l'alarme auprès des voyageurs et de l'ensemble de l'industrie touristique.
\end{abstract}

La tendance de l'heure visant à contrebalancer la production personnelle de GES est le voyage à bilan carbone neutre. Tout le monde semble s'y être converti, des chefs d'entreprise aux vedettes de rock, en passant par les hommes et les femmes politiques et, de plus en plus, les voyageurs ordinaires (Anonyme, 2007 ; Sustainable Travel International, 2007). À l'instar d'un nombre croissant de compagnies, l'industrie du tourisme a emboîté le pas en adoptant une stratégie axée sur le bilan carbone neutre. Petit à petit, conférences, stations de ski, circuits touristiques, services de location de voiture et même jeux olympiques se mettent au diapason, tout comme Expedia et Travelocity qui offrent maintenant des programmes de voyages à bilan carbone neutre (Cyberium, 2007 ; Gouvernement du Canada, 2006; Higgins, 2006).

En substance, le voyage à bilan carbone neutre permet d'atténuer l'impact environnemental de chaque trajet grâce à l'achat de crédits d'émission de carbone, le but ultime étant d'absorber le carbone ${ }^{2}$ présent dans l'atmosphère. Pour qu'un voyage soit à bilan carbone neutre, la quantité de crédits d'émissions de carbone achetés devrait être égale à la quantité utilisée par passager. S'il est vrai que le voyage à bilan carbone neutre constitue un pas important dans l'amélioration de la performance environnementale de l'industrie du tourisme, il n'en demeure pas moins que c'est une solution à court terme qui ne permettra aucunement de renverser la vapeur en ce qui concerne le changement climatique.

Le climat est une ressource importante pour l'industrie touristique. II sera sans aucun doute moins clément dans certaines régions du monde, menacées par les conséquences prévues du réchauffement climatique du globe, telles les inondations, l'érosion côtière et des conditions météorologiques plus extrêmes (Amelung et Viner,
2006; Perry, 2006). En revanche, d'autres destinations tireront profit de la modification du régime climatique, laquelle pourrait favoriser le tourisme.

\section{Le voyage à l'ère de l'écoefficacité}

Selon le Groupe d'experts intergouvernemental sur l'évolution du climat, l'industrie du transport est responsable de $20 \%$ des émissions de GES, $80 \%$ de cette proportion étant attribuables au transport routier et $12 \%$ à l'aviation. Les vols de passagers représentent $2 \%$ à $3 \%$ de l'industrie de l'aviation, mais les données statistiques sur les autres moyens de transport associés au tourisme ne sont pas systématiquement documentées. II est utile de souligner que le transport représente environ $70 \%$ de l'impact environnemental total d'un touriste moyen, le reste étant imputé à la consommation de biens et services divers. Le bilan carbone d'un touriste dépend largement de ses habitudes de consommation ; il est possible de calculer la consommation et la contribution totale de GES de chaque personne et de chaque entreprise (Gössling et al., 2004 ; Becken et Patterson, 2006).

II est par ailleurs très difficile de mesurer l'impact réel sur l'environnement de l'ensemble de l'industrie touristique, celle-ci étant intersectorielle et non traditionnelle. De toute évidence, il existe un écart considérable entre l'empreinte écologique d'un séjour dans un hôtel cinq étoiles alimenté au charbon et celle d'un séjour dans un hôtel semblable alimenté à l'énergie géothermique. De même, une visite au musée aura généralement un impact moindre qu'une excursion à bord d'un bateau à moteur (Becken et Patterson, 2006; Becken et al., 2006 ; Byrnes et Warnken, 2006). Compte tenu des prévisions de croissance annuelle de $4 \%$ et de $5 \%$ jusqu'en 2015 en ce qui concerne le transport aérien de passagers et le tourisme respectivement, la croissance de l'industrie du voyage demeure l'un des plus grands défis du tourisme durable (OMT, 2006 et IATA, 2006). On s'attend notamment à ce que les mouvements aériens doublent d'ici 2020 et qu'ils triplent d'ici 2030 (Boehmer, 2006).

Fort heureusement, l'industrie du tourisme travaille à améliorer son écoefficacité, mais il reste encore beaucoup à faire (Gössling et al., 2004). La Déclaration de Djerba sur le tourisme et le changement climatique de 2003 reconnaît l'importance du rôle du secteur touristique dans la lutte contre l'incidence nuisible du réchauffement de la planète ainsi que le rôle du Protocole de Kyoto (OMT, 2003). Malgré une certaine tendance vers un mode de consommation écologique, 
des recherches récentes indiquent que la plupart des touristes ne sont pas complètement conscients des conséquences qu'ont leurs choix sur l'environnement ni de leur contribution au changement climatique (Gössling et al., 2006).

En dépit de l'augmentation des surtaxes imposées au transport aérien, le coût du voyage ne traduit pas véritablement les coûts réels pour l'environnement. On peut se réjouir des efforts déployés par l'industrie de l'aviation pour appliquer des pratiques davantage écologiques et limiter sa contribution au changement climatique (IATA, 2006 ; Lynes et Dredge, 2006 ; Mak et Chan, 2006). En conséquence, le rendement énergétique des aéronefs s'est amélioré de $70 \%$ au cours des quarante dernières années et on prévoit une amélioration de 20 \% par passager par kilomètre d'ici 2015 (Clayton, 2005 et IPCC, 1999). En 2004 et en 2005 seulement, le rendement énergétique des aéronefs s'est amélioré de $5 \%$; l'Airbus A380 et le Boeing 787 sont maintenant en mesure de transporter 100 passagers à raison de trois litres de carburant par kilomètre, ce qui est tout à fait comparable à la performance de la majorité des voitures à faible consommation d'essence (IATA, 2006). L'amélioration de la conception des aéroports et de la gestion du trafic aérien pourrait également favoriser une diminution du délestage carburant jusqu'à concurrence de $18 \%$ (IPCC, 1999).

Malgré tout, aucun type de transport aérien ne peut encore être considéré comme étant écologique, puisqu'ils exigent tous de brûler des combustibles fossiles. Par ailleurs, les aéronefs émettent de nombreux gaz nocifs dont les effets sont quatre fois plus nuisibles à 30000 pieds qu'au niveau du sol. On compte aujourd'hui près de 12000 aéronefs de passagers en opération à l'échelle mondiale, et la Chine à elle seule devrait avoir besoin de 2900 nouveaux avions au cours des vingt prochaines années (Clayton, 2005). Heureusement, la plupart des transporteurs à faibles coûts utilisent de nouveaux aéronefs (Airline Business, 2007b).

\section{L'industrie du transport aérien et l'échange de droits d'émission de carbone}

L'industrie du tourisme, y compris le secteur de l'aviation, n'est pas (encore) réglementée et n'est donc en aucune façon tenue d'aborder de front les problèmes liés au changement climatique. En outre, les compagnies aériennes bénéficient d'une exemption de taxes sur le carburant pour les vols internationaux. Jusqu'à présent, le projet d'imposer une écotaxe sur les voyages n'a pas eu beaucoup de succès au sein de l'industrie. À la fin de 2006, le Parlement européen a proposé un projet de loi visant l'inclusion de l'industrie aéronautique dans le système d'échange de crédits d'émission de carbone de I'Union européenne. Cette proposition a été accueillie favorablement par quelques compagnies aériennes telles que Virgin Atlantic et British Airways, de même que par l'European Low Fares Airline Association (Becken et Lane, 2006 ; Boehmer, 2006 ; IATA, 2006), mais le secteur global s'y oppose (Airline Business, 2007a).

L'échange de droits d'émission de carbone (ou le commerce de droits de pollution sous forme de quotas de pollution établis par les gouvernements) constituerait un incitatif économique à réduire les GES. On imposerait ainsi à chaque compagnie une limite sur la quantité de dioxyde de carbone qu'elle peut émettre, sous forme de crédits qui lui donneraient le droit de polluer jusqu'à un niveau donné. Celles qui produiraient un taux d'émissions supérieur à leurs droits de pollution auraient à racheter des crédits et celles qui disposeraient de crédits de pollution inutilisés pourraient les revendre. Ce système récompenserait donc les compagnies aériennes qui parviendraient à réduire leurs émissions.

Outre un mécanisme pour un développement propre (MDP) administré par les gouvernements respectifs, le Protocole de Kyoto prévoit un marché officiel du carbone qui régit l'échange de droits d'émission, comme la Bourse de l'Union européenne et celle de Chicago. Un marché de carbone parallèle, volontaire celui-là, a également été instauré pour permettre aux particuliers et aux entreprises de compenser leurs émissions par des contributions financières à des projets de compensation.

Le MDP sert à relier le marché du carbone aux projets de développement durable dans les pays en développement. Ainsi, les nations industrialisées qui ont besoin de réduire leurs émissions de gaz à effet de serre peuvent contribuer au développement durable des pays en développement en investissant dans des projets de compensation qui y sont mis en œuvre. Le MDP a été largement critiqué pour sa tendance à préconiser les projets à grande échelle tels que les épandages contrôlés et les plantations d'arbres en monoculture, lesquels ne profitent pas toujours aux communautés locales. Des problèmes d'administration et d'infrastructure ont également fait surface (Special Report on Carbon..., 2006).

\section{Mesures volontaires pour minimiser les répercussions environnementales des voyages}

Les voyageurs peuvent améliorer leur bilan carbone de deux façons: d'abord en réduisant les émissions directement, ensuite en compensant pour la pollution générée lorsque celle-ci ne peut être ni réduite ni supprimée. Ils peuvent, par exemple, utiliser un mode de transport moins polluant et privilégier les produits et services écologiques ou responsables. D'un point de vue économique, réduire la fréquence des voyages n'est pas souhaitable. D'une façon générale, les déplacements de courte distance alliés à un mode de transport écoefficient sous-tendent le voyage fondé sur le développement durable. On peut aussi améliorer l'écoefficacité à la destination de voyage, en choisissant des produits et services touristiques de façon responsable, en prolongeant la durée du séjour et en achetant davantage de produits écologiques ou responsables (Gössling et al., 2004 ; Responsibletravel.com, 2007).

L'achat volontaire de crédits d'émission de carbone à l'intérieur des programmes de compensation n'étant pas soumis aux exigences administratives du Protocole de Kyoto, les organismes qui vendent des crédits d'émission de carbone dans des projets de compensation prolifèrent. La compensation est généralement mesurée en tonnes de $\mathrm{CO}_{2}$ et, idéalement, les compensations créées doivent êtres facilement vérifiables par un organisme fiable et agréé. Le coût et le choix des projets de compensation des émissions de carbone sont fonction du lieu d'achat (tableau 1). Aujourd'hui, plus de 30 entreprises et organisations vendent des crédits de carbone (Compart, 2007).

Les programmes de compensation des émissions de carbone visent à appuyer des projets variés, notamment le développement 
de l'énergie renouvelable, le développement de la technologie écoénergétique, la destruction des gaz industriels et la séquestration de carbone dans les sols et les forêts. La séquestration du carbone désigne l'augmentation du stock de carbone dans un "réservoir » non atmosphérique. La plupart des compagnies offrent une gamme variée d'options d'investissement dans la technologie écoénergétique ou dans l'achat de " certificats verts " d'énergie renouvelable, telle l'énergie éolienne, solaire, de biomasse ou géothermique.

II n'est pas aisé actuellement d'obtenir des statistiques sur la taille du marché visé, encore moins en ce qui concerne le secteur du tourisme, mais il ne fait aucun doute que la compensation volontaire des émissions de GES connaît un succès grandissant. On peut citer l'exemple de British Airways qui a lancé, en 2005, son propre programme de compensation pour inciter ses clients à contribuer au développement de l'énergie renouvelable (Department of Environment, Food and Rural Affairs, 2005).

\section{Tableau 1}

Estimation des émissions pour un voyage Montréal-Paris et coût de compensation de la pollution produite par l'entremise des projets favorisant le développement de l'énergie renouvelable

\begin{tabular}{l|c|c}
$\begin{array}{l}\text { Voyage aller-retour } \\
\text { Montréal - Paris }\end{array}$ & $\begin{array}{c}\text { Estimation des émissions } \\
\text { de dioxyde de carbone } \\
\text { par personne }\end{array}$ & $\begin{array}{c}\text { Coût de la compensation } \\
\text { en dollars canadiens } \\
\text { investis dans des projets } \\
\text { d'énergie renouvelable }\end{array}$ \\
\hline Carbon Neutral Company & $1200 \mathrm{~kg}$ & 25,38 \\
Climate Care & $1530 \mathrm{~kg}$ & 26,05 \\
Green my Flight & $1502 \mathrm{~kg}$ & 37,57 \\
Offsetters Carbon Neutral Society & $3056 \mathrm{~kg}$ & 57,66 \\
$\begin{array}{l}\text { My Climate (offert par l'entremise } \\
\text { de l'organisme Sustainable }\end{array}$ & $2225 \mathrm{~kg}$ & 81,09 \\
Travel International) & $3800 \mathrm{~kg}$ & 116,28 \\
Atmosfair & $4241 \mathrm{~kg}$ & 163,10 \\
Bonneville Environmental Foundation &
\end{tabular}

Source : les calculs des émissions publiés sur les sites Web des compagnies respectives.

(Calculs et taux de change en vigueur le 19 février 2007.)
A priori, l'application volontaire du principe de bilan carbone neutre présente des avantages non négligeables pour l'industrie du transport aérien. En effet, l'internationalisation du coût environnemental des voyages pourrait permettre aux entreprises de réduire leurs coûts d'exploitation tout en continuant à faire des profits. Une approche volontaire permettrait également d'éviter l'intervention de l'État ainsi que les coûts et les problèmes complexes de conformité y afférents. Qui plus est, en adoptant des pratiques écologiques, les compagnies aériennes véhiculeront une image de compagnie responsable, ce qui pourrait les aider à se démarquer de leurs concurrents, à renforcer le profil de la société et à améliorer leur compétitivité. L'adoption d'une politique écologique pourrait aussi se traduire par l'attraction de nouveaux investisseurs.

\section{Le dédale des projets de compensation}

Le principe de compensation des émissions ne fait pas l'unanimité, ses détracteurs le percevant comme l'attribution du «droit de polluer ». Par ailleurs, compte tenu de leurs désavantages, l'efficacité de certains projets de compensation est discutable. Choisir un projet de compensation n'est donc pas toujours chose facile.

Les plantations en monoculture, particulièrement dans les pays en développement, soulèvent une vive controverse. Les arbres servent au stockage temporaire du carbone dans le cycle normal d'échange de carbone entre les forêts et l'atmosphère; de plus, ils peuvent facilement libérer du carbone dans l'atmosphère lors d'incendies, de changements climatiques, du processus de décomposition naturelle et de la déforestation (Special Report on Carbon..., 2006). La plantation d'arbres est néanmoins près de dix fois moins coûteuse que l'investissement dans la nouvelle technologie (Van Kooten et al., 2004). Cependant, il s'avère que les plantations à grande échelle entraînent la réduction de la biodiversité et sont sources de violence et de conflits relatifs aux terres.
Les projets de plantation d'arbres peuvent créer une biomasse pour la substitution de combustibles fossiles comme l'éthanol et favoriser la création d'emplois (Baral et Guha, 2004). Toutefois, l'investissement dans des projets de développement du biodiésel provenant de produits de l'agriculture comme le sucre ou le maïs soulève des préoccupations en raison du recours au génie génétique, sans compter que la production est généralement associée à l'impact négatif de l'agriculture. En outre, le coût de production du biodiésel varie grandement selon le type de culture et les conditions régionales, et les effets des différents biodiésels sur la réduction des émissions de GES sont loin d'être uniformes. Dans l'ensemble, les investissements dans des projets qui favorisent le développement de l'énergie renouvelable, qu'elle soit solaire, éolienne, hydraulique ou géothermique, tiennent donc le haut du pavé.

\section{Conclusion}

La compensation des émissions se heurte à la critique sur les plans des coûts et de la certification, mais aussi parce qu'elle n'incite pas les consommateurs à utiliser des énergies renouvelables de préférence aux combustibles fossiles. La compensation ne prévoit pas la réglementation des activités polluantes, mais elle doit néanmoins être encouragée comme mesure provisoire.

L'utilisation d'une énergie de substitution issue de ressources renouvelables semble davantage viable et efficace par rapport au coût au fur et à mesure des progrès technologiques. On pourrait ainsi mettre un frein à l'augmentation du taux de carbone atmosphérique et réduire le taux actuel par l'entremise de projets de séquestration visant à rétablir le bilan du carbone de la planète.

Le principe du «pollueur-payeur » dans le contexte du développement durable nécessite que le pollueur fournisse des efforts pour limiter son impact sur l'environnement. Dans le domaine du touris- 
me, cela exige la collaboration des acteurs de l'industrie, mais aussi celle des voyageurs, lesquels devraient adopter des habitudes de consommation plus responsables. Comme le montre le tableau 1, les coûts encourus, bien que variables, demeurent abordables. Mais avant d'exiger des voyageurs qu'ils fassent des choix responsables, il est primordial de les sensibiliser aux conséquences environnementales de leurs choix et aux avantages d'y remédier.

Julianna Priskin est professeure associée au Département d'études urbaines et touristiques de l'Université du Québec à Montréal et chercheure à la Chaire de tourisme Transat de la même Université.

\section{Notes}

Je tiens à remercier Pierre Bellerose, Annelies Hodge et Manfred Fussi pour l'inspiration qu'ils m'ont apportée en plus des discussions passionnées sur le sujet.

1 GES : Le Protocole de Kyoto attribue principalement le réchauffement climatique à six gaz, à savoir le dioxyde de carbone, le méthane, l'oxyde nitreux, les hydrofluorocarbones, les perfluorocarbones et I'hexafluorure de soufre, les incidences sur le réchauffement climatique de la planète différant selon le gaz. Les termes "gaz à effet de serre" et "carbone" sont interchangeables dans le présent article.

2 Un projet de compensation permet de réduire ou d'annuler les émissions de tous les gaz à effet de serre. Par la suite, ces derniers seront convertis en $\mathrm{CO}_{2}$ pour les fins du calcul de crédits de carbone.

\section{Bibliographie}

"Special Report on Carbon Related Issues », New Internationalist, n 391 , juillet 2006.

Airline Business (2007a), "Carbon Storm: Emission Trading Scheme ", Airline Business News, 22 janvier (consulté par le biais de [flightglobal.com]).

Airline Business (2007b), «Time to Carbon Trade », Airline Business, vol. 12, n० 1, p. 9.

Amelung, B., et D. Viner (2006), «Mediterranean Tourism: Exploring the Future with the Tourism Climatic Index", Journal of Sustainable Tourism, vol. 14, n 4, p. 349-366.

Anonyme (2007), "Resorts Nationwide Energize Green Power Program », National Ski Areas Association Journal, vol. 14, n 6, p. 7.

Atmosfair, [www.atmosfair.de], (consulté le 19 février 2007).

Baral, A., et G.S. Guha (2004), «Trees for Carbon Sequestration or Fossil Fuel Substitution: The Issue of Cost vs. Benefit », Biomass and Bioenergy, vol. 27, n० 1, p. 41-55.

Becken, S., et B. Lane (2006), «Air Travel and the Environment, an Interview with Hugh Somerville, Journal of Sustainable Tourism ", vol. 14, n² 2, p. 216-219.

Becken, S., et M. Patterson (2006), «Measuring National Carbon Dioxide Emissions from Tourism as a Key Step towards Achieving Sustainable Tourism ", Journal of Sustainable Tourism, vol. 14, n 4, p. 323-338.

Becken, S., D.G. Simmons, et C. Frampton (2006), «Energy Use Associated with Different Travel Choices », Tourism Management, vol. 24, p. 267-277.

Boehmer, J. (2006), «Virgin Leading the Effort To Reduce $\mathrm{CO}_{2}$ Emissions », [Business Travel news.com], (consulté le 9 octobre 2006).

Bonneville Environmental Foundation, [www.greentagsusa.org], (consulté le 10 février 2007).

Byrnes, T.A., et J. Warnken (2006), "Greenhouse Gas Emissions from Marine Tours: A Case Study of Australian Tour Boat Operators ", Journal of Sustainable Tourism, vol. 14, n³, p. 255-270.
Carbon Neutral Company, [www.carbonneutral.com], (consulté le 19 février 2007).

Clayton, M. (2005), « Jet Pollution: Drawing a Line in the Sky », Trave/wire News, 2 octobre 2005, 3 p.

Climate Care, [www.co2.org], (consulté le 19 février 2007).

Compart, A. (2007), «It Is Not Easy Being Green: Airlines Feel the Heat To Reduce Emissions ", Airlines, 6 mars 2007.

Cyberium (2007), How To Become a Carbon Neutral Organization, [www. cyberium.co.uk], (consulté le 19 février 2007).

Department of Environment, Food and Rural Affairs (2005), News Release: Carbon Offset Scheme Launched, Government of the United Kingdom, 12 septembre 2005.

Gouvernement du Canada (2006), Commuting and Business Travel. Carbon Neutral, [www.fhio-ifppe.gc.ca/default.asp?lang=En\&n=9076E298-1], (consulté le 22 octobre 2006).

Gössling, S., M. Bredberg, A. Randow, et S. Sandström (2006), «Tourist Perceptions of Climate Change: A Study of International Tourist in Zanzibar ». Current Issues in Tourism, vol. 9, nos 4-5, p. 419-435.

Gössling, S., P. Peeters, J.P. Ceron, G. Dubois, T. Patterson, et R.B. Richardson (2004), «The Eco-efficiency of Tourism », Ecological Economics.

Green My Flight, [www.greenmyflight.com], (consulté le 19 février 2007).

Higgins, M. (2006), "Carbon Neutral: Raising the Ante on Eco-tourism », New York Times, 10 décembre.

IPCC: Intergovernmental Panel on Climate Change (1999), Special Report: Aviation and the Global Atmosphere. Summary for Policymakers, Genève, $23 \mathrm{p}$.

IPCC: Intergovernmental Panel on Climate Change (2007), Climate Change 2007: The Physical Science Basis. Summary for Policymakers. Contribution of Working Group I to the Fourth Assessment Report of the Intergovernmental Panel on Climate Change, Genève, $18 \mathrm{p}$.

International Air Transport Association, [www.iata.org], (consulté le 24 septembre 2006).

Lynes, J.K., et D. Dredge (2006), « Going Green: Motivation for Environmental Commitment in the Airline Industry. A Case Study of Scandinavian Airlines ", Journal of Sustainable Tourism, vol. 14, n² 2, p. 116-138.

Mak, B., et W.W. Chan (2006), "Environmental Reporting of Airlines in the Asia Pacific Region ", Journal of Sustainable Tourism, vol. 14, nº 6, p. 618-627.

My Climate via Sustainable Tourism International, [www.sustainabletravel international.org], (consulté le 19 février 2007).

Organisation mondiale du tourisme (2006), Climate Change and Tourism, [www.world-tourism.org], (consulté le 16 novembre 2006).

Organisation mondiale du tourisme (2003), Djerba Declaration on Climate Change and Tourism, Djerba, Tunisia, [www.world-tourism.org/sustai nable/concepts.htm], (consulté le 16 novembre 2006).

Offsetters Carbon Neutral Society, [www.offsetters.com], (consulté le 19 février 2007).

Perry, A. (2006), «Will Predicted Climate Change Compromise the Sustainability of Mediterranean Tourism? ", Journal of Sustainable Tourism, vol. 14, $n^{\circ} 4$, p. 367-375.

Responsibletravel.com (2007), Fly me to the Moon—but Switch Off the TV First, [www.responsibletravel.com], (consulté le 19 février 2007).

Sustainable Travel International (2007), Sustainable Travel International's Responsible Travel Report, vol. 4, nº 10, 13 p.

Van Kooten, G.C., A.J. Eagle, J Manley, et T. Smolak (2004), « How Costly Are Carbon Offsets? A Meta-analysis of Carbon Forest Sinks ", Environmental Science and Policy, nº 7, p. 239-251. 